\title{
Prognostic significance of cardiac magnetic resonance-based markers in patients with hypertrophic cardiomyopathy
}

\author{
Zsofia Dohy ${ }^{1}$ - Liliana Szabo ${ }^{1}$ - Attila Toth ${ }^{1}$. Csilla Czimbalmos ${ }^{1} \cdot$ Rebeka Horvath $^{1}$ - Viktor Horvath ${ }^{1}$. \\ Ferenc Imre Suhai ${ }^{1} \cdot$ Laszlo Geller $^{1} \cdot$ Bela Merkely $^{1} \cdot$ Hajnalka Vago $^{1}$ (I)
}

Received: 31 July 2020 / Accepted: 8 October 2020 / Published online: 8 February 2021

(c) The Author(s) 2021

\begin{abstract}
The prognosis of patients with hypertrophic cardiomyopathy (HCM) varies greatly. Cardiac magnetic resonance (CMR) is the gold standard method for assessing left ventricular (LV) mass and volumes. Myocardial fibrosis can be noninvasively detected using CMR. Moreover, feature-tracking (FT) strain analysis provides information about LV deformation. We aimed to investigate the prognostic significance of standard CMR parameters, myocardial fibrosis, and LV strain parameters in HCM patients. We investigated $187 \mathrm{HCM}$ patients who underwent CMR with late gadolinium enhancement and were followed up. LV mass (LVM) was evaluated with the exclusion and inclusion of the trabeculae and papillary muscles (TPM). Global LV strain parameters and mechanical dispersion (MD) were calculated. Myocardial fibrosis was quantified. The combined endpoint of our study was all-cause mortality, heart transplantation, malignant ventricular arrhythmias and appropriate implantable cardioverter defibrillator (ICD) therapy. The arrhythmia endpoint was malignant ventricular arrhythmias and appropriate ICD therapy. The LVM index (LVMi) was an independent CMR predictor of the combined endpoint independent of the quantification method $(\mathrm{p}<0.01)$. The univariate predictors of the combined endpoint were LVMi, global longitudinal (GLS) and radial strain and longitudinal MD (MDL). The univariate predictors of arrhythmia events included LVMi and myocardial fibrosis. More pronounced LV hypertrophy was associated with impaired GLS and increased MDL. More extensive myocardial fibrosis correlated with impaired GLS $(\mathrm{p}<0.001)$. LVMi was an independent CMR predictor of major events, and myocardial fibrosis predicted arrhythmia events in HCM patients. FT strain analysis provided additional information for risk stratification in HCM patients.
\end{abstract}

Keywords Hypertrophic cardiomyopathy · Cardiac magnetic resonance · Prognosis · Left ventricular hypertrophy · Feature-tracking strain analysis $\cdot$ Myocardial fibrosis

\section{Introduction}

Bela Merkely and Hajnalka Vago have contributed equally to this work.

Zsofia Dohy, Liliana Szabo, Attila Toth, Csilla Czimbalmos, Rebeka Horvath, Viktor Horvath, Ferenc Imre Suhai, Laszlo Geller, Bela Merkely, and Hajnalka Vago takes responsibility for all aspects of the reliability and freedom from bias of the data presented and their discussed interpretation.

Hajnalka Vago

vagoha@gmail.com

1 Heart and Vascular Center, Semmelweis University, 68 Varosmajor St, Budapest 1122, Hungary
Hypertrophic cardiomyopathy (HCM) is a primary myocardial disease that is characterized by substantial heterogeneity in phenotypic expression, clinical course, and overall prognosis. HCM is one of the most common causes of sudden cardiac death (SCD) in young patients; another complication of HCM is progressive heart failure [1, 2]. The typical pathological features of HCM include myocyte disarray, smallvessel disease, and myocardial fibrosis, which usually have a patchy mid-myocardial distribution in the hypertrophic segments [3]. The structural abnormalities in HCM cause alterations in left ventricular (LV) mechanics [4]. The relation of the structural and mechanical characteristics to the clinical outcomes of patients with HCM has been partially explored. 
According to the current European and American guidelines on HCM, the SCD risk stratification is based on age, family history of SCD, maximal LV wall thickness, left atrial diameter, LV outflow tract gradient, previous unexplained syncope and the occurrence of non-sustained ventricular tachycardia [5, 6], however, data in the literature suggest an additional prognostic role of the detection and quantification of myocardial fibrosis using cardiac magnetic resonance (CMR) examination [7, 8].

LV hypertrophy is usually measured using maximal end-diastolic wall thickness, and the diagnosis of HCM and SCD risk stratification are based on this parameter. However, LV mass (LVM) may more accurately describe hypertrophy. CMR-based quantification of LVM can be carried out with different evaluation methods. During conventional evaluation, the endocardial layer is detected along the inner border of the compact myocardium, resulting in the trabeculae and papillary muscle (TPM) being included in the ventricular cavity. Threshold-based methods define the endocardial surface based on the different signal intensities of blood and myocardium; thus, the TPM are measured as part of the ventricular mass [9]. There is no consensus on which evaluation method is more reliable in the case of HCM [10].

Strain analysis is a useful and reliable method for the assessment of global and regional myocardial function. Global LV strain parameters are considered a sensitive and early marker of LV dysfunction. The damage in different myocardium layers affects certain directions of deformation during LV contraction that can be detected using strain analysis even with normal ejection fraction (EF). Because of myocardial disarray and fibrosis, myocardial contraction in $\mathrm{HCM}$ is heterogeneous. Mechanical dispersion (MD) assessed by strain measurement reflects this heterogeneous contraction [11]. Strain analysis can help in the diagnosis and may guide the prognosis of patients with HCM; however, these findings are based on speckle tracking echocardiography $[4,12,13]$. Feature-tracking (FT) CMR has been validated for strain measurement using standard cine CMR images [14]. However, limited data are available on whether CMR-based strain analysis has incremental prognostic value in patients with $\operatorname{HCM}[15,16]$.

Despite the advantages of the detailed evaluation of HCM patients, including strain analysis and myocardial fibrosis quantification, there is a lack of comprehensive studies with large study populations that integrate the prognostic significance of all of these data provided by CMR. Therefore, we conducted a study with the aim of investigating the prognostic significance of the following CMR parameters in one model: (1) different LV hypertrophy parameters such as LVM calculated using conventional and threshold-based methods and maximal end-diastolic wall thickness; (2) LV functional parameters such as ejection fraction (EF) and
FT-CMR-based global LV strain values and MD; and (3) amount of myocardial fibrosis.

\section{Methods}

\section{Study population}

We enrolled consecutive patients who underwent CMR examination in our tertiary referral centre between January 2009 and March 2019. The patients were followed up in this retrospective longitudinal observational study. Our inclusion criteria were the unequivocal diagnosis of HCM and lack of confounding comorbidities, such as untreated hypertension, significant aortic stenosis, previous myocardial infarction and prior surgical myectomy or percutaneous transluminal septal myocardial ablation. We excluded patients if there were no available clinical follow-up data, they did not receive contrast agent, or if the strain analysis was not properly feasible (Fig. 1).

Informed consent was obtained from each patient. Ethical approval was obtained from the Hungarian National Institute of Pharmacy and Nutrition (OGYEI/29,174-4/2019), and this study was performed in accordance with the ethical standards in the 1964 Declaration of Helsinki and its later amendments.

\section{CMR protocol}

CMR examinations were conducted with a $1.5 \mathrm{~T}$ magnetic resonance (MR) scanner (Achieva, Philips Medical Systems) using a 5-channel cardiac coil. Retrospectively gated balanced steady-state free precession (bSSFP) cine images were acquired in 2-chamber, 4-chamber and LV outflow tract views. Additionally, short-axis (SA) images with full coverage of the LV were obtained. A bolus of gadobutrol $(0.15 \mathrm{mmol} / \mathrm{kg})$ was injected at a rate of $2-3 \mathrm{ml} / \mathrm{s}$ through an antecubital intravenous line. Late gadolinium enhancement (LGE) images were acquired using a segmented inversion recovery sequence with additional phase-sensitive reconstructions in the same views used for cine images 10-20 min after contrast administration.

\section{Image analysis}

CMR data were analysed using Medis Suite 3.1 software (Medis Medical Imaging Software, Leiden, The Netherlands). The left ventricular ejection fraction (LVEF), volumes and LVM were quantified using conventional and threshold-based methods. During conventional contouring, the endo- and epicardial layers were manually traced along the inner and outer borders of the compact myocardium. For TPM quantification, a thresholding algorithm was used 
Fig. 1 Study flow chart

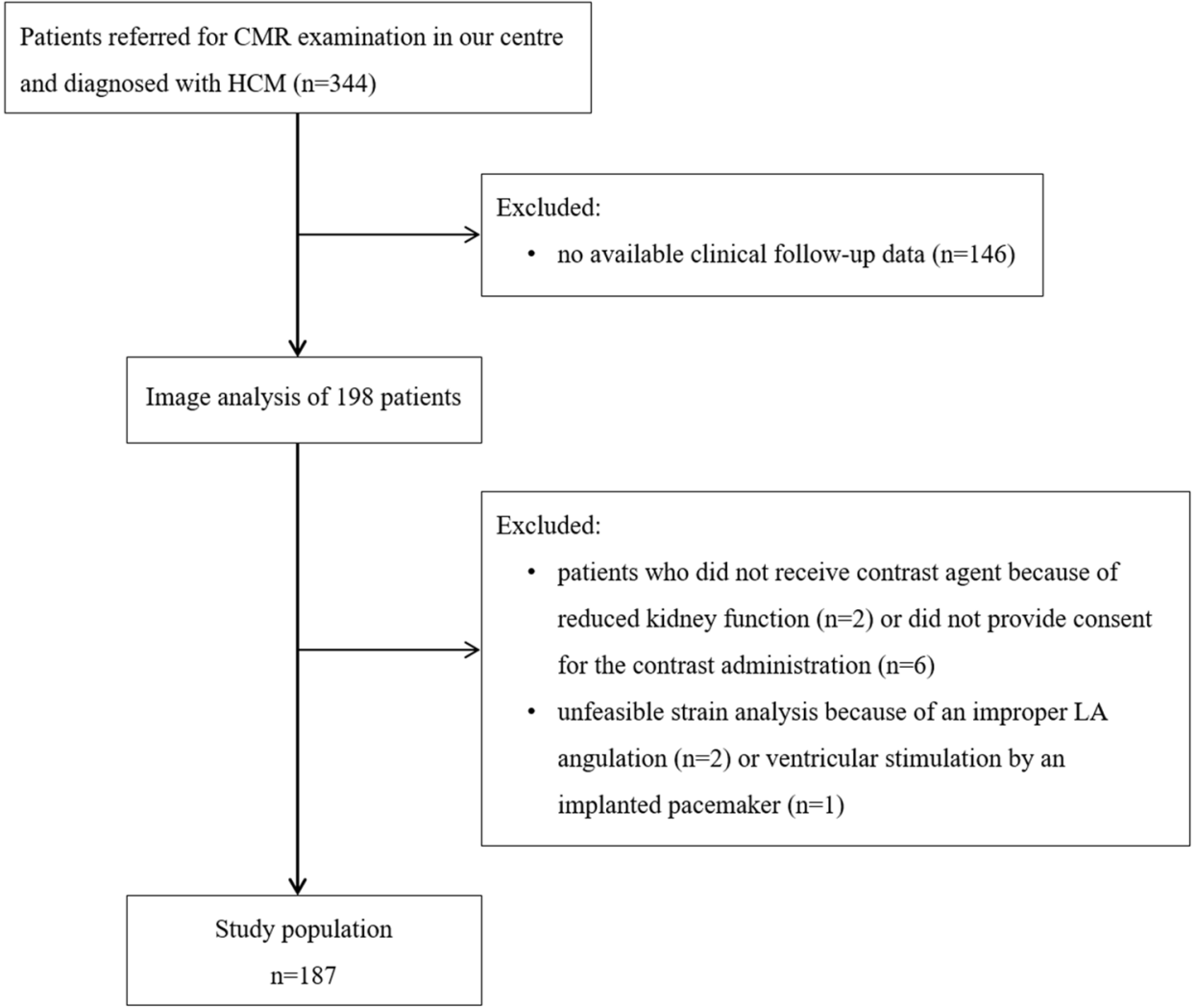

(MassK 7.8, Medis, Leiden, The Netherlands), which distinguishes between blood and myocardium based on their different signal intensities (Fig. 2). We used the same enddiastolic and end-systolic phases for the threshold-based quantification. LV volumes, LVM and TPM were standardized to the body surface area (BSA). Maximal end-diastolic wall thickness measurements were taken in an SA slice perpendicular to the myocardial centre line, excluding trabeculation. On LGE images, myocardial fibrosis was quantified at a grey-scale threshold of 5 standard deviations (SDs) above the mean signal intensity for normal myocardium (Fig. 2). LV strain analysis was obtained with the feature-tracking application of the MedisSuite: QStrain module. Endocardial contour detection was manually performed on long-axis (LA) and SA cine images in end-systolic and end-diastolic phases. Global longitudinal (GLS), circumferential (GCS) and radial (GRS) LV strain parameters were measured. For global dyssynchrony measurement, MD was assessed, which was defined as the SD of the time-to-peak circumferential (MDC) and longitudinal (MDL) strain of the LV segments expressed as percentage of the cardiac cycle.

\section{Endpoint and clinical follow-up}

The clinical follow-up was based on the medical records and the National Health Insurance Fund of Hungary record database, which includes up-to-date information on the date

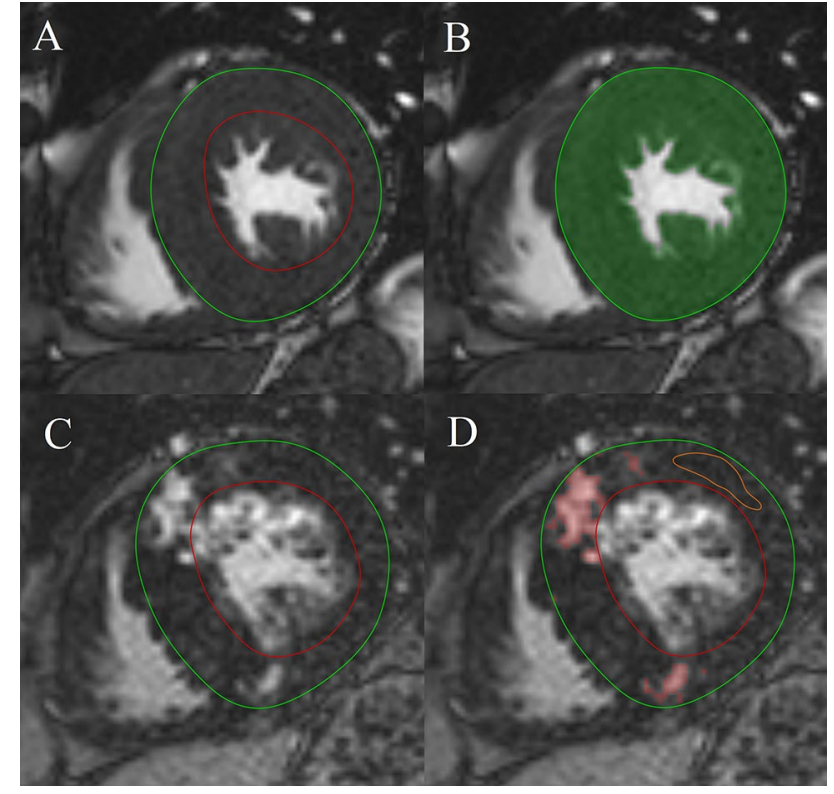

Fig. 2 CMR images of a patient with septal HCM. a, b bSSFP cine short-axis image in the end-diastolic phase. Measurement of LV mass with conventional (a) and threshold-based (b) methods. c, d Delayed contrast enhancement images in the short-axis plane. d Quantification of myocardial fibrosis at $5 \mathrm{SD}$ 
of death. A combined endpoint and an arrhythmia endpoint were analysed. The combined endpoint included all-cause mortality, heart transplantation, and malignant ventricular arrhythmia or appropriate implantable cardioverter defibrillator (ICD) therapy. The arrhythmia endpoint included malignant ventricular arrhythmia and appropriate ICD therapy.

\section{Statistical analysis}

Continuous data are expressed as the mean \pm SD. The normal distribution of data was investigated with the Kolmogorov-Smirnov test. The characteristics of groups were compared with an independent t-test or Mann-Whitney test, as appropriate. The correlation between continuous variables was calculated with Spearman's correlation analysis. The prognostic value of CMR parameters was assessed with univariate and multivariate Cox proportional hazard regression analyses with the enter selection method. Variables with $\mathrm{p}<0.05$ in univariate analyses were candidates for multivariate analyses. Multicollinearity was measured with the variance inflation factor (VIF). Highly correlated predictors (VIF > 2.5) were removed from the multivariate model. Receiver operating characteristic (ROC) curve analysis was performed to identify optimal cut-off values. Univariate associations of time variables with major events were visualized using Kaplan-Meier curves and compared by the log-rank test. Differences were considered statistically significant when $\mathrm{p}<0.05$. All analyses were performed by using MedCalc software (version 17.9.5).

\section{Results}

\section{Patient characteristics}

The demographic and CMR characteristics of the study population are summarized in Table 1. The patients had the following symptoms: syncope (19\%), chest pain $(41 \%)$, dyspnoea (39\%), and palpitation (36\%). Three patients were examined after aborted SCD. Based on echocardiography and electrocardiography (ECG) findings, HCM was the referral diagnosis in $96 \%$ of the cases, while in the remaining $4 \%$ of the patients, the referral diagnosis was amyloidosis, left ventricular noncompaction, arrhythmogenic cardiomyopathy, anomalous origin of a coronary artery, previous myocardial infarction or undefined structural abnormality. Echocardiography described obstructive HCM in $34 \%$ of the cases.

\section{CMR characteristics}

The majority of the study population (147 patients) had a normal LVEF (57-77\%), two patients had a supra-normal LVEF (>77\%), 36 patients had a mildly reduced LVEF (41-56\%), and two patients had a moderately reduced LVEF (30-40\%). The most common form of HCM was asymmetric
Table 1 Demographic and CMR characteristics of the study population. Comparison of the parameters of patients with and without combined or arrhythmia endpoints

\begin{tabular}{|c|c|c|c|c|c|c|}
\hline & \multicolumn{3}{|c|}{ Combined endpoint } & \multicolumn{3}{|c|}{ Arrhythmia endpoint } \\
\hline & Yes & No & $\mathrm{p}$ & Yes & No & $\mathrm{p}$ \\
\hline Number of patients & 34 & 153 & & 12 & 168 & \\
\hline Male & $13(38 \%)$ & $86(56 \%)$ & 0.06 & $5(42 \%)$ & $92(55 \%)$ & 0.38 \\
\hline Age (y) & $47.8 \pm 20.9$ & $46.8 \pm 17.9$ & 0.52 & $36.0 \pm 22.0$ & $47.4 \pm 17.9$ & 0.09 \\
\hline $\operatorname{BSA}\left(m^{2}\right)$ & $1.81 \pm 0.26$ & $1.91 \pm 0.25$ & 0.06 & $1.70 \pm 0.37$ & $1.90 \pm 0.25$ & 0.07 \\
\hline LVEF (\%) & $62.4 \pm 6.9$ & $62.9 \pm 7.7$ & 0.74 & $60.2 \pm 8.0$ & $62.9 \pm 7.6$ & 0.26 \\
\hline LVESVi $\left(\mathrm{ml} / \mathrm{m}^{2}\right)$ & $34.9 \pm 13.4$ & $33.2 \pm 10.8$ & 0.51 & $38.2 \pm 16.2$ & $33.3 \pm 11.0$ & 0.24 \\
\hline LVEDVi $\left(\mathrm{ml} / \mathrm{m}^{2}\right)$ & $91.7 \pm 26.4$ & $88.1 \pm 17.0$ & 0.65 & $93.8 \pm 28.2$ & $88.4 \pm 18.2$ & 0.65 \\
\hline LVSVi (ml/m²) & $56.6 \pm 15.5$ & $55.4 \pm 10.9$ & 0.95 & $55.2 \pm 14.6$ & $55.5 \pm 11.7$ & 0.67 \\
\hline $\operatorname{LVMi}_{\text {conv }}\left(\mathrm{g} / \mathrm{m}^{2}\right)$ & $114.9 \pm 52.1$ & $88.0 \pm 31.2$ & $<0.001$ & $126.2 \pm 56.5$ & $90.4 \pm 35.0$ & $<0.01$ \\
\hline $\operatorname{LVMi}_{\mathrm{TB}}\left(\mathrm{g} / \mathrm{m}^{2}\right)$ & $142.2 \pm 67.5$ & $113.0 \pm 37.4$ & $<0.01$ & $160.8 \pm 75.2$ & $115.5 \pm 42.1$ & $<0.01$ \\
\hline TPMi (g/m²) & $29.0 \pm 15.2$ & $24.9 \pm 8.4$ & 0.17 & $34.0 \pm 19.7$ & $25.1 \pm 9.0$ & $<0.05$ \\
\hline Maximal wall thickness (mm) & $22.2 \pm 5.7$ & $20.6 \pm 5.7$ & 0.14 & $23.0 \pm 6.0$ & $20.9 \pm 5.7$ & 0.20 \\
\hline Myocardial fibrosis (g) & $20.9 \pm 18.6$ & $16.6 \pm 21.4$ & $<0.05$ & $29.3 \pm 22.9$ & $16.4 \pm 21.0$ & $<0.05$ \\
\hline Myocardial fibrosis (\%) & $9.8 \pm 7.4$ & $8.4 \pm 8.9$ & 0.12 & $13.1 \pm 8.7$ & $8.2 \pm 8.7$ & $<0.05$ \\
\hline GLS (\%) & $-21.2 \pm 6.2$ & $-22.9 \pm 5.4$ & 0.20 & $-20.6 \pm 6.9$ & $-22.7 \pm 5.4$ & 0.27 \\
\hline GCS $(\%)$ & $-40.3 \pm 8.6$ & $-40.2 \pm 7.5$ & 0.90 & $-39.1 \pm 9.0$ & $-40.0 \pm 7.5$ & 0.68 \\
\hline GRS (\%) & $76.6 \pm 22.0$ & $83.4 \pm 22.5$ & 0.11 & $74.8 \pm 21.1$ & $82.4 \pm 22.6$ & 0.26 \\
\hline $\operatorname{MDL}(\%)$ & $17.7 \pm 4.6$ & $16.4 \pm 5.2$ & 0.17 & $17.7 \pm 5.7$ & $16.5 \pm 5.1$ & 0.44 \\
\hline $\operatorname{MDC}(\%)$ & $8.5 \pm 4.7$ & $7.1 \pm 3.8$ & 0.10 & $9.3 \pm 5.0$ & $7.2 \pm 3.8$ & 0.16 \\
\hline
\end{tabular}


hypertrophy with a septal or an anterior distribution, which was found in 161 patients (81.5\%). There were 27 (13.5\%) patients with apical HCM, 7 (3.5\%) patients with concentric HCM and three (1.5\%) patients with midventricular HCM. Myocardial fibrosis was detected in $90.6 \%$ of patients. More extensive myocardial fibrosis was associated with a higher LVM index $(\mathrm{LVMi})(\mathrm{p}<0.0001, \mathrm{r}=0.495)$ and higher maximal end-diastolic wall thickness $(\mathrm{p}<0.0001$, $r=0.44$ ). Impaired GLS correlated with a higher LVMi and more extensive myocardial fibrosis. Higher maximal enddiastolic wall thickness correlated with higher MDL, referring to more pronounced global LV dyssynchrony. LVEF did not correlate with LV hypertrophy or with the amount of myocardial fibrosis (Fig. 3).

\section{Clinical outcome}

During the follow-up (3.8 \pm 2.4 years), 20 patients died, and the cause of death was known in 11 patients. One patient died because of brain cancer, cardiovascular death was obvious in 10 cases, and one of them had SCD. In the case of 9 patients, the cause of death was unknown, and these patients were not included in the statistical analyses regarding arrhythmia events. Six patients underwent heart transplantation. One patient had aborted SCD during the followup period. ICD implantation occurred in 52 patients (48 as primary prevention), appropriate ICD therapy was detected in 9 patients (6 DC shock, 3 antitachycardia pacing), and one patient had electrical storm. The three patients who were examined after aborted SCD had appropriate ICD therapy during the follow-up.

\section{The prognostic value of CMR}

The patients who reached the combined endpoint had higher LVMi both with the conventional ( $\left.\mathrm{LVMi}_{\text {conv }}\right)$ and threshold-based $\left(\mathrm{LVMi}_{\mathrm{TB}}\right)$ evaluation methods. The patients with arrhythmia events had higher $\mathrm{LVMi}_{\text {conv }}$ and $\mathrm{LVMi}_{\mathrm{TB}}$, higher TPM index (TPMi) and more extended myocardial fibrosis (Table 1).

In the apical HCM group, the endpoint of our study was detected in only one patient who had heart transplantation; however, statistically, there was no difference in the prognosis of the different morphological types of HCM.

$\mathrm{LVMi}_{\text {conv, }} \mathrm{LVMi}_{\mathrm{TB}}$, GLS, GRS and MDL were significant univariate predictors of the combined endpoint. In the multivariate models, LVMi was an independent predictor of the combined endpoint $(\mathrm{p}<0.01)$. We investigated the prognostic factors of arrhythmia events, and we found that LV endsystolic volume index (ESVi), LVEF, $\mathrm{LVMi}_{\text {conv, }} \mathrm{LVMi}_{\mathrm{TB}}$, TPMi and myocardial fibrosis were significant univariate predictors of arrhythmia events (Table 2).

Using ROC analysis, we calculated different LVMi cutoffs regarding the combined endpoint: the LVMi cut-off for males with the conventional evaluation method was $108 \mathrm{~g} / \mathrm{m}^{2}$, and it was $128 \mathrm{~g} / \mathrm{m}^{2}$ with the threshold-based method. The LVMi cut-off for females with the conventional evaluation method was $86 \mathrm{~g} / \mathrm{m}^{2}$, and it was $107 \mathrm{~g} /$
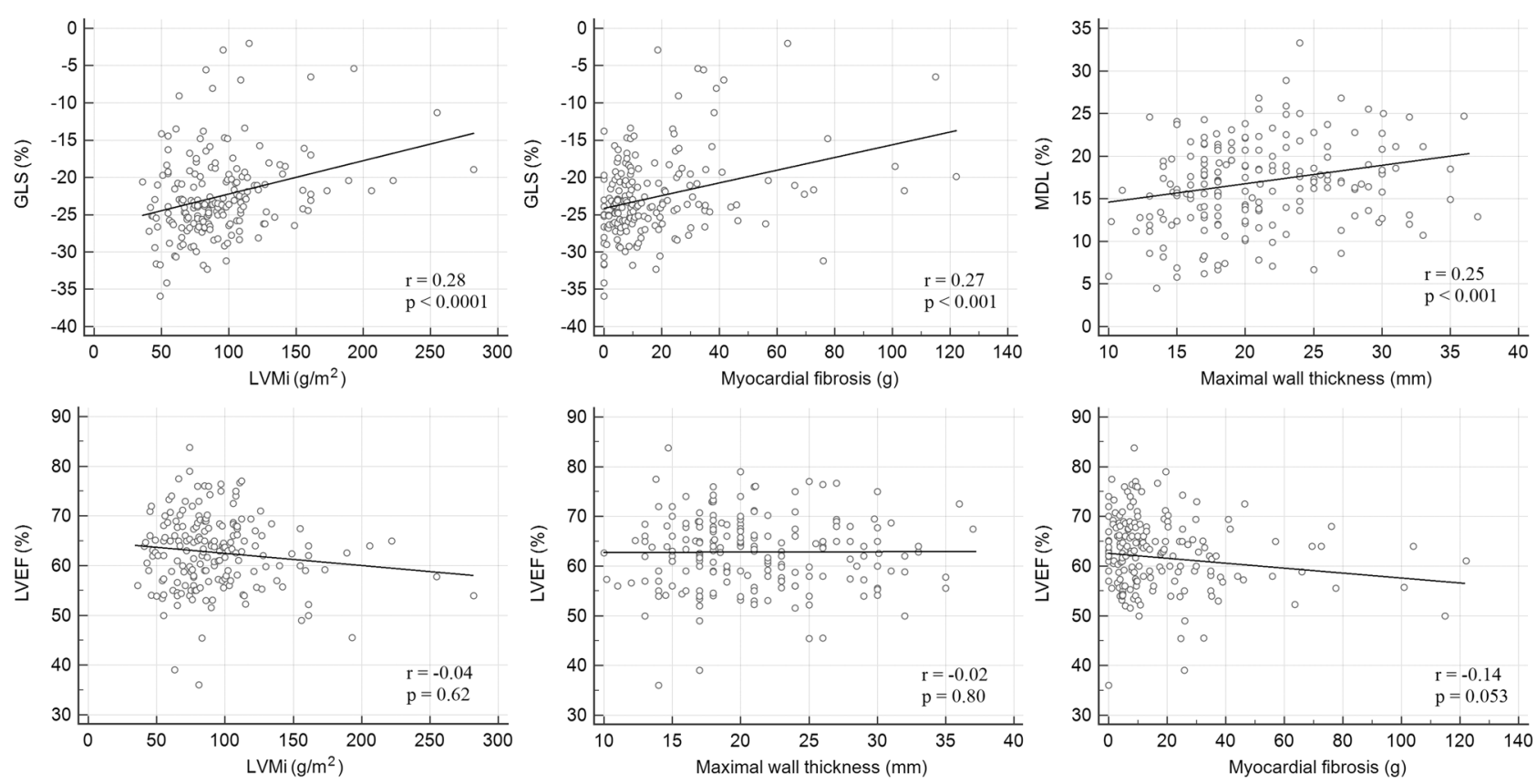

Fig. 3 Correlation between LV functional parameters and LV hypertrophy and myocardial fibrosis (Spearman's correlation) 
Table 2 Predictors of the combined and arrhythmia endpoints assessed with univariate and multivariate Cox proportional hazard regression analyses

\begin{tabular}{|c|c|c|c|c|c|c|}
\hline & \multicolumn{2}{|c|}{ Univariate analysis } & \multicolumn{2}{|c|}{$\begin{array}{l}\text { Multivariate analysis with } \\
\text { LVMi }_{\text {Conv }}\end{array}$} & \multicolumn{2}{|c|}{$\begin{array}{l}\text { Multivariate analysis with } \\
\mathrm{LVMi}_{\mathrm{TB}}\end{array}$} \\
\hline & $\mathrm{p}$ & $\operatorname{HR}[95 \% \mathrm{CI}]$ & $\mathrm{p}$ & $\mathrm{HR}[95 \% \mathrm{CI}]$ & $\mathrm{p}$ & $\mathrm{HR}[95 \% \mathrm{CI}]$ \\
\hline \multicolumn{7}{|l|}{ Combined endpoint } \\
\hline Age & 0.21 & $1.01[0.99$ to 1.03$]$ & & & & \\
\hline Female gender & 0.06 & $1.93[0.97$ to 3.87$]$ & & & & \\
\hline LVEDVi & 0.56 & $1.01[0.99$ to 1.02$]$ & & & & \\
\hline LVESVi & 0.13 & $1.02[0.99$ to 1.05$]$ & & & & \\
\hline LVSVi & 0.57 & $0.99[0.96$ to 1.02$]$ & & & & \\
\hline LVEF & 0.051 & $0.95[0.91$ to 1.00$]$ & & & & \\
\hline $\mathrm{LVMi}_{\text {Conv }}$ & 0.002 & $1.01[1.003$ to 1.02$]$ & 0.011 & $1.01[1.00$ to 1.02$]$ & & \\
\hline $\mathrm{LVMi}_{\mathrm{TB}}$ & 0.005 & $1.01[1.002$ to 1.01$]$ & & & 0.02 & $1.01[1.00$ to 1.01$]$ \\
\hline TPMi & 0.07 & $1.02[1.00$ to 1.04$]$ & & & & \\
\hline Maximal end-diastolic wall thickness & 0.90 & $1.004[0.95$ to 1.06$]$ & & & & \\
\hline Myocardial fibrosis (\%) & 0.42 & $1.01[0.98$ to 1.05$]$ & & & & \\
\hline Myocardial fibrosis (g) & 0.51 & $1.005[0.99$ to 1.02$]$ & & & & \\
\hline GLS & 0.02 & $1.08[1.01$ to 1.15$]$ & 0.27 & $1.04[0.97$ to 1.12$]$ & 0.26 & $1.04[0.97$ to 1.12$]$ \\
\hline GCS & 0.81 & 1.01 [1.01 to 1.05$]$ & & & & \\
\hline GRS & 0.048 & $0.98[0.97$ to 0.99$]$ & & & & \\
\hline MDL & 0.048 & $1.07[1.00$ to 1.14$]$ & 0.12 & 1.06 [0.99 to 1.13$]$ & 0.13 & $1.06[0.98$ to 1.13$]$ \\
\hline MDC & 0.06 & 1.08 [0.99 to 1.17$]$ & & & & \\
\hline \multicolumn{7}{|l|}{ Arrhythmia endpoint } \\
\hline Age & 0.19 & $0.98[0.94$ to 1.01$]$ & & & & \\
\hline Female gender & 0.34 & $1.76[0.55$ to 5.59$]$ & & & & \\
\hline LVEDVi & 0.54 & $1.01[0.98$ to 1.04$]$ & & & & \\
\hline LVESVi & 0.08 & $1.04[1.00$ to 1.08$]$ & & & & \\
\hline LVSVi & 0.39 & $0.98[0.93$ to 1.03$]$ & & & & \\
\hline LVEF & 0.03 & 0.91 [0.84 to 0.99$]$ & 0.15 & 0.93 [0.84 to 1.03$]$ & 0.13 & $0.92[0.83$ to 1.06$]$ \\
\hline $\mathrm{LVMi}_{\mathrm{Conv}}$ & 0.01 & $1.01[1.00$ to 1.02$]$ & 0.28 & 1.01 [0.99 to 1.03$]$ & & \\
\hline $\mathrm{LVMi}_{\mathrm{TB}}$ & 0.009 & $1.01[1.00$ to 1.02$]$ & & & 0.21 & $1.01[0.99$ to 1.03$]$ \\
\hline TPMi & 0.02 & $1.03[1.00$ to 1.06$]$ & 0.71 & $0.99[0.92$ to 1.06$]$ & 0.50 & $0.97[0.89$ to 1.06$]$ \\
\hline Maximal end-diastolic wall thickness & 0.76 & $1.02[0.92$ to 1.12$]$ & & & & \\
\hline Myocardial fibrosis (\%) & 0.03 & $1.05[1.01$ to 1.09$]$ & 0.14 & $1.03[0.99$ to 1.08$]$ & 0.15 & $1.03[0.99$ to 1.08$]$ \\
\hline Myocardial fibrosis (g) & 0.07 & $1.02[1.00$ to 1.04$]$ & & & & \\
\hline GLS & 0.053 & $1.11[1.00$ to 1.22$]$ & & & & \\
\hline GCS & 0.58 & $1.02[0.95$ to 1.10$]$ & & & & \\
\hline GRS & 0.15 & $0.98[0.95$ to 1.01$]$ & & & & \\
\hline MDL & 0.22 & $1.07[0.96$ to 1.19$]$ & & & & \\
\hline MDC & 0.09 & $1.12[0.98$ to 1.27$]$ & & & & \\
\hline
\end{tabular}

In the analysis of the predictors of the combined endpoint, LVMiconv, LVMiTB, GLS, GRS and MDL were significant in the univariate analyses. Multicollinearity was measured with the variance inflation factor (VIF), and GLS and GRS were highly correlated predictors (VIF $>2.5$ ); therefore, GRS was removed from the model. $\mathrm{LVMi}_{\text {conv }}$ and $\mathrm{LVMi}_{\mathrm{TB}}$ were significant predictors of the combined endpoint in the multivariate analysis. In the analysis of the predictors of the arrhythmia endpoint, the significant univariate predictors were LVESVi, LVEF, LVMi, TPMi and myocardial fibrosis 
Fig. 4 LVMi cut-off for males and females with ROC analysis regarding major events. Eventfree survival of patients divided by LVMi cut-off (Kaplan-Meier curves)
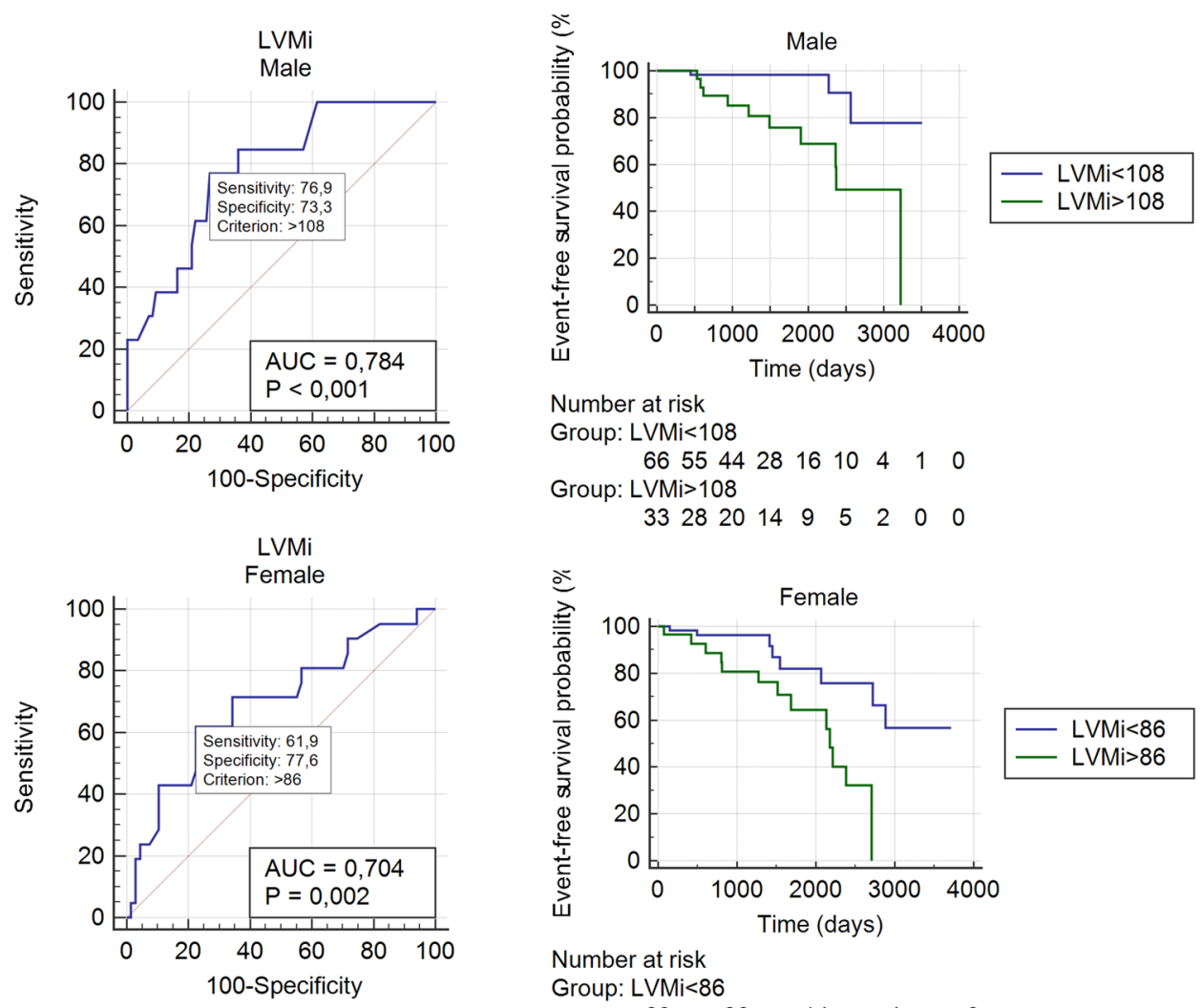

$\mathrm{m}^{2}$ with the threshold-based method. The patients with an LVMi less than the cut-off value had significantly better prognosis (Fig. 4).

\section{Discussion}

The main findings of our single-centre study with a relatively large HCM population are as follows:

1. LVMi was an independent CMR predictor of major events (mortality, heart transplantation, malignant ventricular arrhythmia or appropriate ICD therapy) independent of the LVMi quantification method.

2. The univariate predictors of major events were LVMi, GLS, GRS and MDL. The univariate predictors of arrhythmia events were LVESVi, LVEF, LVMi, TPMi and myocardial fibrosis.

3. More pronounced LV hypertrophy was associated with impaired GLS and increased MDL. More extended myocardial fibrosis correlated with impaired GLS. However, LVEF showed no correlation with the degree of LV hypertrophy or with the extent of myocardial fibrosis.
The primary role for CMR in patients with HCM is the clarification of the diagnosis. The prognostic significance of CMR has been under discussion in this patient population; therefore, the current guidelines on HCM do not contain CMR examination in the risk stratification [6], or recommend that CMR be considered in selected patients with HCM for whom risk remains borderline after documentation of conventional risk factors [5]. Several studies have demonstrated that patients with more extensive myocardial fibrosis have a higher risk for malignant ventricular arrhythmias [7, $8,17,18]$. Myocardial fibrosis in HCM consists of varying degrees of both replacement fibrosis and interstitial fibrosis. In our study, both diffuse and definite contrast enhancement were considered indications of myocardial fibrosis, which explains the high proportion (90.6\%) of patients with myocardial fibrosis. Based on a previous study, the 5SD quantification method showed the strongest correlation with the histopathological assessment of total fibrosis (the sum of replacement and interstitial fibrosis) [19]. In our prognostic model, the extent of myocardial fibrosis (assessed with 5SD) was analysed. Our results are consistent with the supposition of the prognostic role of myocardial fibrosis; in our patient population, myocardial fibrosis was a univariate predictor of malignant ventricular arrhythmias. 
Conventionally, maximal end-diastolic wall thickness is used to describe LV hypertrophy and to estimate SCD risk in HCM [20-22]. However, data in the literature regarding the prognostic significance of maximal wall thickness have been controversial [23]. LVM is a more robust measure of the total burden of LV hypertrophy than a single measurement of the maximal wall thickness. Spiewak et al. stated that the maximal wall thickness does not reflect the degree of LV hypertrophy in patients with HCM, as patients with the same wall thickness may have substantial differences in LVM [24]. Olovitto et al. found that markedly increased LVMi was more sensitive in predicting outcome, whereas maximal end-diastolic wall thickness $>30 \mathrm{~mm}$ was more specific in HCM patients [25]. CMR examination provides the most accurate and reproducible information about LVM, as CMR-based LVM measurements are free of cardiac geometric assumptions [26]. There are different evaluation methods of LVM that either include TPM or exclude it from the mass. We found that higher LVMi predicted poor clinical outcome independent of the evaluation method; nevertheless, the LVMi cut-off values regarding major events depended on the evaluation method and sex. In our patient population, maximal end-diastolic wall thickness was not a predictor of major events.

Myocardial strain analyses provide accurate information about global and regional LV function. Strain by speckle tracking echocardiography has been increasingly applied as a sensitive and early marker of LV dysfunction in different cardiomyopathies. Previous studies have demonstrated the relation of echocardiography-based strain parameters to structural alterations and clinical outcomes in patients with HCM $[4,12,27]$. Hinojar et al. investigated the prognostic implication of global strain parameters assessed with FTCMR in 74 patients with HCM. They found that impaired global LV strain values were associated with all-cause mortality and heart failure events [15]. MD was not investigated in their study; however, a previous study demonstrated the prognostic role of MD evaluated with FT-CMR in patients after ST-segment elevation myocardial infarction [28]. In our study, GLS, GRS and MDL were univariate predictors of major events. In patients with more pronounced LV hypertrophy, we found increased global LV dyssynchrony and impaired longitudinal contraction, while LVEF did not correlate with the degree of hypertrophy. These results suggested that the FT strain analysis provides important additional information for the detection of LV dysfunction and for risk stratification in $\mathrm{HCM}$ patients.

\section{Study limitations}

The limitations of our study are that it was a single-centre study, which might limit the generalizability of our conclusions. Although our study was designed to represent a real-world population, due to the retrospective nature of the study, limited clinical data were available for some patients; therefore, these patients were excluded from the analyses. Myocardial T1 and T2 mapping and myocardial extracellular volume evaluation were not available. In the vast majority of the patients, no genetic testing was performed.

\section{Conclusion}

Our results show that LVMi is an independent CMR predictor of major events and that myocardial fibrosis predicts arrhythmia events in HCM patients. Furthermore, FT strain analysis provides additional information for the detection of LV dysfunction and for risk stratification in HCM patients.

Author contributions Zsofia Dohy, Liliana Szabo, Attila Toth, Csilla Czimbalmos, Ferenc Imre Suhai, Laszlo Geller, Bela Merkely and Hajnalka Vago takes responsibility for all aspects of the reliability and freedom from bias of the data presented and their discussed interpretation.

Funding Open Access funding provided by Semmelweis University. Supported by the ÚNKP-19-3-I New National Excellence Program of the Ministry for Innovation and Technology. This project was supported by a grant from the National Research, Development and Innovation Office (NKFIH) of Hungary (K 120277). This study was supported by the National Research, Development and Innovation Office of Hungary (NKFIA; NVKP_16-1-2016-0017 National Heart Program). The research was financed by the Thematic Excellence Programme (Tématerületi Kiválósági Program, 2020-4.1.1.-TKP2020) of the Ministry for Innovation and Technology in Hungary, within the framework of the Therapeutic Development and Bioimaging thematic programme of the Semmelweis University.

\section{Compliance with ethical standards}

Conflict of interest The authors declare that they have no conflict of interest.

Open Access This article is licensed under a Creative Commons Attribution 4.0 International License, which permits use, sharing, adaptation, distribution and reproduction in any medium or format, as long as you give appropriate credit to the original author(s) and the source, provide a link to the Creative Commons licence, and indicate if changes were made. The images or other third party material in this article are included in the article's Creative Commons licence, unless indicated otherwise in a credit line to the material. If material is not included in the article's Creative Commons licence and your intended use is not permitted by statutory regulation or exceeds the permitted use, you will need to obtain permission directly from the copyright holder. To view a copy of this licence, visit http://creativecommons.org/licenses/by/4.0/.

\section{References}

1. Maron BJ (2018) Clinical course and management of hypertrophic cardiomyopathy. N Engl J Med 379(7):655-668. https:// doi.org/10.1056/NEJMra1710575 
2. Maron BJ, Haas TS, Ahluwalia A, Murphy CJ, Garberich RF (2016) Demographics and epidemiology of sudden deaths in young competitive athletes: from the United States National Registry. Am J Med 129(11):1170-1177. https://doi.org/10.1016/j. amjmed.2016.02.031

3. Varnava AM, Elliott PM, Sharma S, McKenna WJ, Davies MJ (2000) Hypertrophic cardiomyopathy: the interrelation of disarray, fibrosis, and small vessel disease. Heart (British Cardiac Society) 84(5):476-482. https://doi.org/10.1136/heart.84.5.476

4. Haland TF, Almaas VM, Hasselberg NE, Saberniak J, Leren IS, Hopp E, Edvardsen T, Haugaa KH (2016) Strain echocardiography is related to fibrosis and ventricular arrhythmias in hypertrophic cardiomyopathy. Eur Heart J Cardiovasc Imaging 17(6):613-621. https://doi.org/10.1093/ehjci/jew005

5. Gersh BJ, Maron BJ, Bonow RO, Dearani JA, Fifer MA, Link MS, Naidu SS, Nishimura RA, Ommen SR, Rakowski H, Seidman CE, Towbin JA, Udelson JE, Yancy CW (2011) 2011 ACCF/AHA Guideline for the diagnosis and treatment of hypertrophic cardiomyopathy: a report of the American College of Cardiology Foundation/American Heart Association Task Force on Practice Guidelines. Developed in collaboration with the American Association for Thoracic Surgery, American Society of Echocardiography, American Society of Nuclear Cardiology, Heart Failure Society of America, Heart Rhythm Society, Society for Cardiovascular Angiography and Interventions, and Society of Thoracic Surgeons. J Am Coll Cardiol 58(25):e212e260. https://doi.org/10.1016/j.jacc.2011.06.011

6. Elliott PM, Anastasakis A, Borger MA, Borggrefe M, Cecchi F, Charron P, Hagege AA, Lafont A, Limongelli G, Mahrholdt H, McKenna WJ, Mogensen J, Nihoyannopoulos P, Nistri S, Pieper PG, Pieske B, Rapezzi C, Rutten FH, Tillmanns C, Watkins H (2014) 2014 ESC Guidelines on diagnosis and management of hypertrophic cardiomyopathy: the Task Force for the Diagnosis and Management of Hypertrophic Cardiomyopathy of the European Society of Cardiology (ESC). Eur Heart J 35(39):27332779. https://doi.org/10.1093/eurheartj/ehu284

7. Doesch C, Tulumen E, Akin I, Rudic B, Kuschyk J, El-Battrawy I, Becher T, Budjan J, Smakic A, Schoenberg SO, Borggrefe M, Papavassiliu T (2017) Incremental benefit of late gadolinium cardiac magnetic resonance imaging for risk stratification in patients with hypertrophic cardiomyopathy. Sci Rep 7(1):6336. https://doi.org/10.1038/s41598-017-06533-0

8. Weng Z, Yao J, Chan RH, He J, Yang X, Zhou Y, He Y (2016) Prognostic value of LGE-CMR in HCM: a meta-analysis. JACC Cardiovasc Imaging 9(12):1392-1402. https://doi.org/10.1016/j. jemg.2016.02.031

9. Csecs I, Czimbalmos C, Suhai FI, Mikle R, Mirzahosseini A, Dohy Z, Szucs A, Kiss AR, Simor T, Toth A, Merkely B, Vago $\mathrm{H}$ (2018) Left and right ventricular parameters corrected with threshold-based quantification method in a normal cohort analyzed by three independent observers with various trainingdegree. Int J Cardiovasc Imaging 34(7):1127-1133. https://doi. org/10.1007/s10554-018-1322-4

10. Gommans DH, Bakker J, Cramer GE, Verheugt FW, Brouwer MA, Kofflard MJ (2016) Impact of the papillary muscles on cardiac magnetic resonance image analysis of important left ventricular parameters in hypertrophic cardiomyopathy. Neth Heart J 24(5):326-331. https://doi.org/10.1007/s12471-016-0805-y

11. Schnell F, Matelot D, Daudin M, Kervio G, Mabo P, Carre F, Donal E (2017) Mechanical dispersion by strain echocardiography: a novel tool to diagnose hypertrophic cardiomyopathy in athletes. J Am Soc Echocardiogr 30(3):251-261. https://doi. org/10.1016/j.echo.2016.11.013

12. Saito M, Okayama H, Yoshii T, Higashi H, Morioka H, Hiasa G, Sumimoto T, Inaba S, Nishimura K, Inoue $K$, Ogimoto A, Shigematsu Y, Hamada M, Higaki J (2012) Clinical significance of global two-dimensional strain as a surrogate parameter of myocardial fibrosis and cardiac events in patients with hypertrophic cardiomyopathy. Eur Heart J Cardiovasc Imaging 13(7):617-623. https://doi.org/10.1093/ejechocard/jer318

13. Vergé MP, Cochet H, Reynaud A, Morlon L, Peyrou J, Vincent C, Rooryck C, Ritter P, Lafitte S, Réant P (2018) Characterization of hypertrophic cardiomyopathy according to global, regional, and multi-layer longitudinal strain analysis, and prediction of sudden cardiac death. Int J Cardiovasc Imaging 34(7):1091-1098. https://doi.org/10.1007/s10554-018-1323-3

14. Pedrizzetti G, Claus P, Kilner PJ, Nagel E (2016) Principles of cardiovascular magnetic resonance feature tracking and echocardiographic speckle tracking for informed clinical use. J Cardiovasc Magn Reson 18(1):51. https://doi.org/10.1186/s1296 8-016-0269-7

15. Hinojar R, Fernandez-Golfin C, Gonzalez-Gomez A, Rincon LM, Plaza-Martin M, Casas E, Garcia-Martin A, FernandezMendez MA, Esteban A, Nacher JJJ, Zamorano JL (2017) Prognostic implications of global myocardial mechanics in hypertrophic cardiomyopathy by cardiovascular magnetic resonance feature tracking. Relations to left ventricular hypertrophy and fibrosis. Int J Cardiol 249:467-472. https://doi.org/10.1016/j. ijcard.2017.07.087

16. Smith BM, Dorfman AL, Yu S, Russell MW, Agarwal PP, Ghadimi Mahani M, Lu JC (2014) Relation of strain by feature tracking and clinical outcome in children, adolescents, and young adults with hypertrophic cardiomyopathy. Am J Cardiol 114(8):1275-1280. https://doi.org/10.1016/j.amjca rd.2014.07.051

17. He D, Ye M, Zhang L, Jiang B (2018) Prognostic significance of late gadolinium enhancement on cardiac magnetic resonance in patients with hypertrophic cardiomyopathy. Heart Lung 47(2):122-126. https://doi.org/10.1016/j.hrtlng.2017.10.008

18. Green JJ, Berger JS, Kramer CM, Salerno M (2012) Prognostic value of late gadolinium enhancement in clinical outcomes for hypertrophic cardiomyopathy. JACC Cardiovasc Imaging 5(4):370-377. https://doi.org/10.1016/j.jcmg.2011.11.021

19. Moravsky G, Ofek E, Rakowski H, Butany J, Williams L, RalphEdwards A, Wintersperger BJ, Crean A (2013) Myocardial fibrosis in hypertrophic cardiomyopathy: accurate reflection of histopathological findings by CMR. JACC Cardiovasc Imaging 6(5):587-596. https://doi.org/10.1016/j.jcmg.2012.09.018

20. O’Mahony C, Jichi F, Pavlou M, Monserrat L, Anastasakis A, Rapezzi C, Biagini E, Gimeno JR, Limongelli G, McKenna WJ, Omar RZ, Elliott PM (2014) A novel clinical risk prediction model for sudden cardiac death in hypertrophic cardiomyopathy (HCM risk-SCD). Eur Heart J 35(30):2010-2020. https://doi. org/10.1093/eurheartj/eht439

21. Spirito P, Bellone P, Harris KM, Bernabo P, Bruzzi P, Maron BJ (2000) Magnitude of left ventricular hypertrophy and risk of sudden death in hypertrophic cardiomyopathy. N Engl J Med 342(24):1778-1785. https://doi.org/10.1056/nejm20000615342 2403

22. Elliott PM, Gimeno Blanes JR, Mahon NG, Poloniecki JD, McKenna WJ (2001) Relation between severity of left-ventricular hypertrophy and prognosis in patients with hypertrophic cardiomyopathy. Lancet (London, England) 357(9254):420-424. https://doi.org/10.1016/s0140-6736(00)04005-8

23. Olivotto I, Gistri R, Petrone P, Pedemonte E, Vargiu D, Cecchi F (2003) Maximum left ventricular thickness and risk of sudden death in patients with hypertrophic cardiomyopathy. J Am Coll Cardiol 41(2):315-321. https://doi.org/10.1016/s0735 -1097(02)02713-4

24. Spiewak M, Chojnowska L, Małek LA, Miłosz B, Petryka J, Zabicka M, Kłopotowski M, Dabrowski M, Miśko J, Ruzyłło W (2010) Comparison between maximal left ventricular wall 
thickness and left ventricular mass in patients with hypertrophic cardiomyopathy. Kardiologia Polska 68(7):763-768

25. Olivotto I, Maron MS, Autore C, Lesser JR, Rega L, Casolo G, De Santis M, Quarta G, Nistri S, Cecchi F, Salton CJ, Udelson JE, Manning WJ, Maron BJ (2008) Assessment and significance of left ventricular mass by cardiovascular magnetic resonance in hypertrophic cardiomyopathy. J Am Coll Cardiol 52(7):559566. https://doi.org/10.1016/j.jacc.2008.04.047

26. Armstrong AC, Gidding S, Gjesdal O, Wu C, Bluemke DA, Lima JA (2012) LV mass assessed by echocardiography and CMR, cardiovascular outcomes, and medical practice. JACC Cardiovasc Imaging 5(8):837-848. https://doi.org/10.1016/j. jcmg.2012.06.003

27. Reant P, Mirabel M, Lloyd G, Peyrou J, Lopez Ayala JM, Dickie S, Bulluck H, Captur G, Rosmini S, Guttmann O, Demetrescu
C, Pantazis A, Tome-Esteban M, Moon JC, Lafitte S, McKenna WJ (2016) Global longitudinal strain is associated with heart failure outcomes in hypertrophic cardiomyopathy. Heart (British Cardiac Society) 102(10):741-747. https://doi.org/10.1136/ heartjnl-2015-308576

28. Muser D, Tioni C, Shah R, Selvanayagam JB, Nucifora G (2017) Prevalence, correlates, and prognostic relevance of myocardial mechanical dispersion as assessed by feature-tracking cardiac magnetic resonance after a first ST-segment elevation myocardial infarction. Am J Cardiol 120(4):527-533. https://doi. org/10.1016/j.amjcard.2017.05.019

Publisher's Note Springer Nature remains neutral with regard to jurisdictional claims in published maps and institutional affiliations. 